\title{
The SUMO Protease Verloren Regulates Dendrite and Axon Targeting in Olfactory Projection Neurons
}

\author{
Daniela Berdnik, Vincenzo Favaloro, and Liqun Luo \\ Howard Hughes Medical Institute, Department of Biology, Stanford University, Stanford, California 94305
}

\begin{abstract}
Sumoylation is a post-translational modification regulating numerous biological processes. Small ubiquitin-like modifier (SUMO) proteases are required for the maturation and deconjugation of SUMO proteins, thereby either promoting or reverting sumoylation to modify protein function. Here, we show a novel role for a predicted SUMO protease, Verloren (Velo), during projection neuron (PN) target selection in the Drosophila olfactory system. PNs target their dendrites to specific glomeruli within the antennal lobe $(\mathrm{AL})$ and their axons stereotypically into higher brain centers. We uncovered mutations in velo that disrupt PN targeting specificity. PN dendrites that normally target to a particular dorsolateral glomerulus instead mistarget to incorrect glomeruli within the $\mathrm{AL}$ or to brain regions outside the AL. velo mutant axons also display defects in arborization. These phenotypes are rescued by postmitotic expression of Velo in PNs but not by a catalytic domain mutant of Velo. Two other SUMO proteases, DmUlp1 and CG12717, can partially compensate for the function of Velo in PN dendrite targeting. Additionally, mutations in SUMO and lesswright (which encodes a SUMO conjugating enzyme) similarly disrupt PN targeting, confirming that sumoylation is required for neuronal target selection. Finally, genetic interaction studies suggest that Velo acts in SUMO deconjugation rather than in maturation. Our study provides the first in vivo evidence for a specific role of a SUMO protease during neuronal target selection that can be dissociated from its functions in neuronal proliferation and survival.
\end{abstract}

\section{Introduction}

The stereotyped organization of the Drosophila olfactory system provides a model for studying wiring specificity. Each olfactory projection neuron $(\mathrm{PN})$ targets its dendrites specifically to one of 50 glomeruli in the antennal lobe and forms synaptic connections with a specific class of olfactory receptor neurons (Jefferis et al., 2001). Subsequently, each PN axon projects stereotypically to higher brain centers (Marin et al., 2002; Wong et al., 2002; Jefferis et al., 2007). Previous studies have identified several molecules, including transmembrane receptors and transcription factors, acting cellautonomously to regulate $\mathrm{PN}$ dendrite targeting specificity (Komiyama et al., 2003, 2007; Komiyama and Luo, 2007; Hong et al., 2009). However, it has been suggested that misregulation of post-translational modifications may also lead to defects in PN targeting (Tea et al., 2010). In a genetic screen for molecules that regulate PN dendrite wiring specificity, we discovered a previ-

\footnotetext{
Received Dec. 16, 2010; revised April 4, 2012; accepted April 13, 2012.

Author contributions: D.B. and L.L. designed research; D.B. and V.F. performed research; D.B. contributed unpublished reagents/analytic tools; D.B. analyzed data; D.B. and L.L. wrote the paper.

This work was supported by a Long-Term Human Frontier Science Program Postdoctoral Fellowship (D.B.) and a grant from NIH (R01-DC005982 to L.L.). L.L. is an HHMl investigator. We thank members of the Luo laboratory, especially Tim Mosca and Joy Tea, for helpful comments on the manuscript, M. L. Shu for generating the phylogenetic tree (in Figure 4A), D. Luginbuhl for generating transgenic flies, M. Smith for reagents, and the Bloomington, Harvard (Exelixis Collection), and Szeged Stock Centers for fly stocks.

This article is freely available online through the J Neurosci Open Choice option.

Correspondence should be addressed to Liqun Luo at the above address. E-mail: Iluo@stanford.edu.

D. Berdnik's present address: Department of Neurology and Neurological Sciences, Stanford University School of Medicine, Stanford, CA 94305.

DOI:10.1523/JNEUROSCI.6574-10.2012

Copyright $\odot 2012$ the authors $\quad 0270-6474 / 12 / 328331-10 \$ 15.00 / 0$
}

ously uncharacterized Small Ubiquitin-like Modifier (SUMO) protease that we named Verloren (Velo).

Sumoylation is a reversible post-translational modification that occurs in numerous cellular proteins to inhibit, modify or enable protein-protein interactions, thereby modulating protein localization and function. The functional consequences of SUMO attachment vary depending on the substrate and cell type. The majority of identified sumoylated proteins are either localized to the nucleus or involved in the nuclear trafficking of cytosolic proteins (GeissFriedlander and Melchior, 2007). SUMO conjugation is a highly dynamic process and can be rapidly reversed by SUMO proteases. The same family of proteases is also responsible for the maturation of newly synthesized SUMO precursors. SUMO proteases are highly conserved across species and are evolutionary divided into two branches: the yeast ubiquitin-like protein-specific protease 1 (Ulp1) and Ulp2-related proteases (Hay, 2007). Yeast Ulp1 possesses a hydrolase activity required for SUMO maturation and an isopeptidase activity essential for SUMO deconjugation (Li and Hochstrasser, 1999). Ulp2 possesses only the isopeptidase activity ( $\mathrm{Li}$ and Hochstrasser, 2000).

Although sumoylation controls many basic cellular processes like transcriptional regulation, chromatin organization, replication and repair, it is also a key determinant in many neuronal processes (Martin et al., 2007; Scheschonka et al., 2007). Sumoylation plays a role during neuronal development and function. For example, desumoylation of the Monocyte Enhancer Factor 2A (MEF2A) transcription factor leads to MEF2A activation and the inhibition of synapse formation (Shalizi et al., 2006). In addition, SUMO attachment of presynaptic proteins can negatively regulate glutamate release from nerve terminals, thereby control- 
ling neuronal activity (Feligioni et al., 2009). Sumoylation is not restricted to the nucleus but can also occur in the cytoplasm and at the membrane. Furthermore, sumoylation has been implicated in several neurodegenerative diseases, such as in the exacerbation of neurodegeneration in a Huntingtin's disease model (Steffan et al., 2004; Dorval and Fraser, 2007).

Starting from a forward genetic screen, our study reveals an in vivo function for the SUMO protease Velo in controlling neuronal targeting and morphogenesis. Furthermore, our genetic interaction studies suggest that Velo regulates PN target selection via the deconjugation of SUMO protein(s).

\section{Materials and Methods}

Fly stocks. The insertions LL05207, LL05209 and e01260 in velo originate from two piggyBac collections (Thibault et al., 2004; Schuldiner et al., 2008). Information for all other mutant alleles used can be found in Flybase (http://flybase.bio.indiana.edu). Mosaic analysis with a repressible cell marker (MARCM) was performed as previously described using Gal4-GH146 (Wu and Luo, 2006a).

Generation of phylogenetic tree. The phylogenetic tree in Figure $4 A$ displays the relationship between budding yeast $(\mathrm{Sc})$, human $(\mathrm{Hs})$, and Drosophila $(D m)$ homologs of SUMO protease proteins. The number shown at each branch displays the confidence in that branch, as generated by bootstrapping analysis. Every protein in the tree shares the $\mathrm{C} 48$ peptidase catalytic domain. Its corresponding sequence was isolated, aligned using Clustal W multiple protein sequence alignment, and compared. PHYLIP (PHYLogeny Inference Package) v3.69 was used to generate the tree through bootstrapping analysis. This requires using the SEQBOOT program to generate bootstrapped datasets, the PROTPARS program to estimate trees for each bootstrapped dataset based on the parsimony method, and the CONSENSE program to find the clades at each branch based on bootstrap majority probability. The output of CONSENSE is then modified using the RETREE program to root the tree with HsSENP8/NEDP1 yielding the final tree. All programs in the PHYLIP package are freely available online (http://evolution.genetics.washington.edu/phylip/getme.html).

Plasmid and transgene construction. To generate UAS-velo-HA (long), a 5502 base pair (bp) fragment was amplified from a full-length cDNA (LD13895) as a template using the following primers $\left(5^{\prime}-3^{\prime}\right)$ : CACCATGGAG CAGTATATTGTGCCCCTG and CGGCTCTATCTTCCGGATCTTCAG.

To generate UAS-DmUlp1-HA, a 4540 bp fragment was amplified using a full-length cDNA (GH15225) as a template and the following primers $\left(5^{\prime}-3^{\prime}\right)$ : CACCATGTCGCTGCCTCCCGAGGAC and CTGCCACAACTTTCCGTCGGCGATTTC. To generate UAS-CG12717-HA, a $2044 \mathrm{bp}$ fragment was amplified using genomic DNA from $w^{1118}$ flies as a template (no introns are present in CG12717) and the following primers $\left(5^{\prime}-3^{\prime}\right)$ : CACCATGGATCGCAAAGAAACTG and TTTGAGTGTATTCCTTCTCCTCGG. We found that the published sequence of the cDNA clone IP12935 for CG12717 omits a T after nucleotide 37 following the transcriptional start site, yielding an out-of-frame protein. Therefore, we used genomic wild-type DNA as a template to generate this construct.

All PCR products were subcloned into pENTR-D/TOPO (Invitrogen) and recombined into pTWH (Gateway Collection, Drosophila Genomics Resource Center) using the Gateway LR clonase II enzyme mix (Invitrogen). The UAS-velo-HA \#108L (long), UAS-DmUlp1-HA \#5.1, and UAS-CG12717-HA \#4 transgenes, all inserted on the second chromosome and generated by standard injection techniques, were used for rescue experiments.

For Velo expression in Drosophila S2 cells, VeloWT and VeloC $>\mathrm{S}$ cDNAs were recombined from pENTR-D/TOPO into pAWH (Gateway Collection, Drosophila Genomics Resource Center) by LR reaction.

In vitro mutagenesis. In vitro mutagenesis was performed according to manufacturer's instructions (QuikChange Site-Directed Mutagenesis Kit, Stratagene) to generate a point mutation in the velo transgene to generate UAS-velo ${ }^{C>S}$-HA using the primers: GAACAACTTCACCGATAGCGGC CTGTATCTGCTGC and GCAGCAGATACAGGCCGCTATCGGTGAA GTTGTTC. These primers introduce a change from codon TGC to AGC at amino acid 1624 of the long velo transcript resulting in a cysteine to serine change, disrupting the catalytic domain of Velo. A UAS-velo ${ }^{C>S}$-HA \#1.2 insertion on the third chromosome was used for rescue experiments.

FLP-mediated excision to produce CG12717 mutants. The deletion mutant CG12717 $7^{\text {ex\#6 }}$ was generated by FLP-mediated excision described by Parks et al. (2004) using the flanking P-element $\mathrm{P}(\mathrm{XP})$ d05069 and the piggyBac insertion $\mathrm{pBac}(\mathrm{RB}) e 01706 a$. 40 single white females were screened by PCR to confirm the deletion and determine its extent using the primers: AATGATTCGCAGTGGAAGGCT (XP 5' plus, left) and TG CATTTGCCTTTCGCCTTAT (RB 3'plus, right) for the hybrid PCR and CAAATTTCAAAGCGGACTATCG and AGATCCATGTGTTTGCGC TCG to measure the deletion size. CG12717 $7^{\text {ex\# }}$ mutants are homozygous viable and bear a $\sim 2200$ bp deletion that removes the entire open reading frame.

SUMO imprecise P-element excision. The P-element $\mathrm{P}($ lacW $) 2(l) S H 0182$ was mapped by inverse PCR $10 \mathrm{bp}$ upstream of the transcriptional start of the SUMO gene and used to perform an imprecise excision. 100 single white males were screened by PCR to determine the extent of genomic deletions with the primers: GATTCCCTTTGCCATTGAGTACCC and ACTGTGGC CAGAAGTTCAAGGTGG or TATGGTGCTGAGTCATGGTGAGAC. $S U M O^{\text {ex77 }}$ mutants are homozygous lethal and bear a $\sim 1200$ bp deletion that removes the entire open reading frame.

Immunostainings. Confocal images were taken on a LSM510 confocal microscope (Zeiss). Fly brains were dissected, fixed and stained as described previously (Wu and Luo, 2006b). Primary antibody information: rat-anti-mCD8 1:100 (Invitrogen Caltag MCD0800), mouse nc82 1:40 (DSHB \#nc82; E. Buchner, University of Würzburg, Würzburg, Germany), rabbit anti-HA 1:100 (Abcam) and mouse anti-HA 1:500 (gift from K. Wehner, Stanford University, Stanford, CA).

Cell culture, transfections, and Western blotting. Drosophila S2 cells (Invitrogen) were cultured at $25^{\circ} \mathrm{C}$ in Schneider's insect medium (Sigma) supplemented with $10 \%$ heat-inactivated fetal bovine serum (Invitrogen). Transfections were performed on cells growing in 6-well plates, using the Effectene transfection reagent (QIAGEN). $72 \mathrm{~h}$ after transfection a mix on PMSF, NEM (Sigma-Aldrich) and cOmplete, an EDTA-free Protease Inhibitor Cocktail (Roche) were added to the medium. Cells were harvested and lysed in $2 \times$ SDS sample buffer. For Western blot analysis, the lysates were separated on a NuPAGE 3-8\% Tris-acetate gel (Invitrogen) and blotted on nitrocellulose membrane (Protran). Blocking and antibody incubations were performed at room temperature with $7 \% \mathrm{w} / \mathrm{v}$ skimmed milk (Bio-Rad) in $1 \times$ PBS, $0.02 \%$ Tween. Incubation times for primary antibodies were $1 \mathrm{~h}$ and for secondary antibodies $45 \mathrm{~min}$. The following primary antibodies were used: mouse anti- $\alpha$-Tubulin (Sigma-Aldrich, DM1A, 1:1000), mouse anti-HA (12CA5, 1:1000), and mouse anti-GFP (Clontech, \#632375, 1:5000). HRP-conjugated secondary antibodies (Jackson ImmunoResearch) were used at 1:10,000. Blots were developed using the SuperSignal West Femto Maximum Sensitivity Substrate (ThermoScientific) and imaged by ChemiDoc XRS + System and Image Lab Version 2.0.1 software (Bio-Rad).

\section{Results}

\section{verloren is required for $\mathrm{PN}$ dendrite targeting}

To identify genes essential for dendrite targeting in Drosophila olfactory projection neurons (PNs), we performed a MARCMbased mosaic forward genetic screen using a piggyBac transposon collection (Schuldiner et al., 2008). We found that the insertions LL05207 and LL05209, integrated in the first and fifth intron of the long transcript of $C G 10107$, respectively (Fig. $1 A$ ), exhibited PN dendrite targeting defects. Additionally, a third allele, pBac(RB)e01260 (Thibault et al., 2004) which is inserted in the fifth intron of CG10107 (Fig. 1A), also displayed similar PN dendrite targeting defects. All three alleles were homozygous lethal and fail to complement each other (data not shown).

The MARCM technique (Lee and Luo, 1999) allows the visualization and genetic manipulation of PNs in neuroblast and singlecell clones in an otherwise heterozygous animal. We used the postmitotic driver Gal4-GH146 (Stocker et al., 1997) and MARCM 

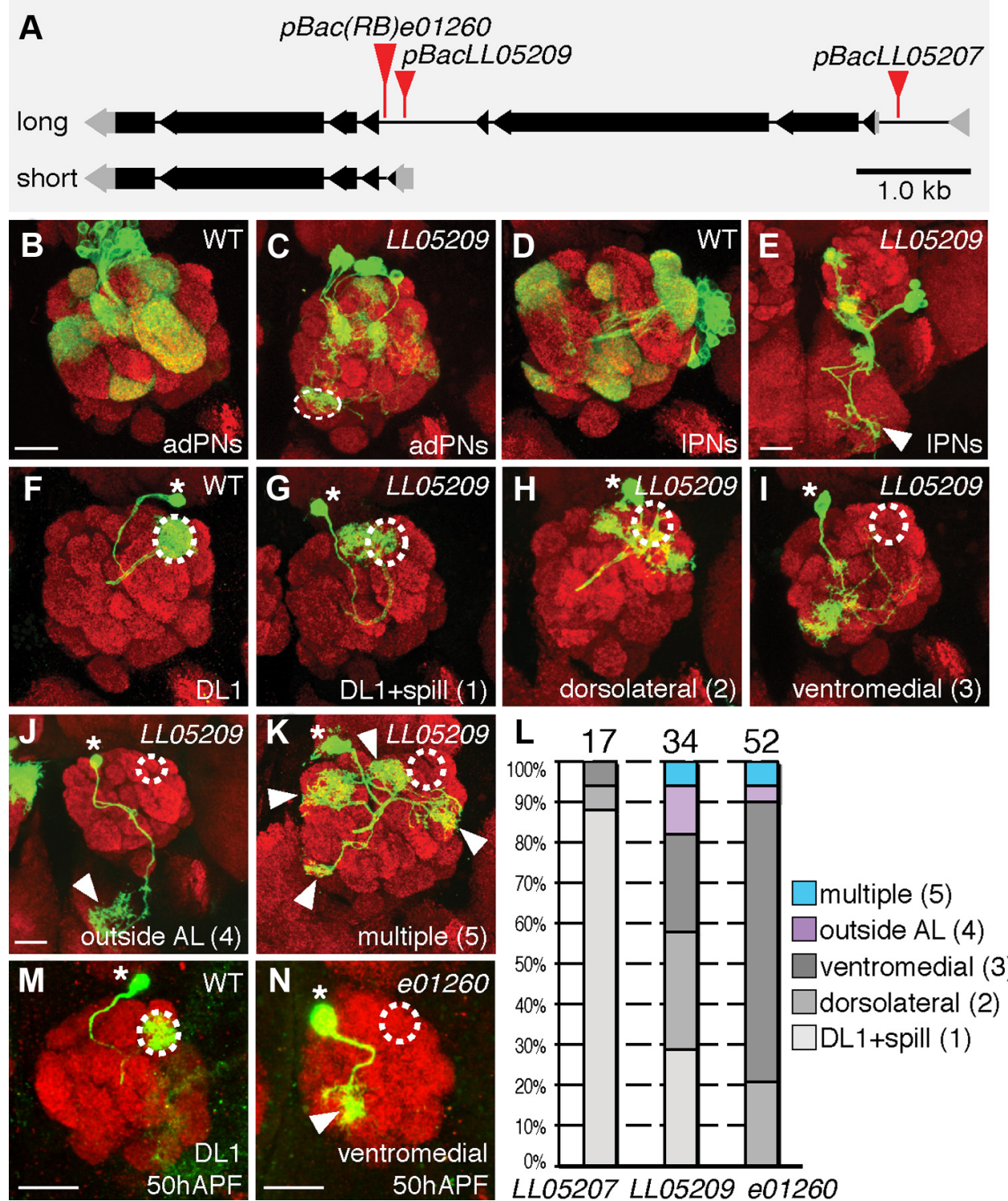

Figure 1. Velo is required cell-autonomously for correct glomerular choice of projection neuron dendrites. $A$, Schematic of the velo gene locus showing a long (5852 bp (G10107-RA) and short (2540 bp (G10107-RB) velo transcript with the piggyBac (pBac) insertions (red triangles) used in this study. A third velo transcript (5867 bp (G10107-RC; data not shown here) differs from the long transcript by 15 bp in the 5'UTR. pBacLL05207 and pBacLL05209 insertions originate from a previously described pBac collection and are inserted 490 bp upstream from the translational start in the first intron of the long transcript and $30 \mathrm{bp}$ upstream from the translational start of the short transcript, respectively (Schuldiner et al., 2008). pBac(RB)e01260 originates from the Exelixis collection (Thibault et al., 2004) and is inserted 33 bp upstream from the second exon in the first intron of the short transcript. Exons are shown as black, UTRs as gray bars, and introns as lines. $\boldsymbol{B}$, WT anterodorsal projection neurons (adPNs) and $\boldsymbol{D}$, lateral projection neurons (IPNs) target a stereotyped set of glomeruli. $\boldsymbol{C}, \boldsymbol{E}$, velo mutant adPNs and IPNs exhibit reduced cell numbers, their dendrites are misorganize, innervating either incorrect glomeruli within (dashed circle in $\boldsymbol{C}$ ) or targets outside the AL (arrowhead in $\boldsymbol{E}$ ). $\boldsymbol{F}$, WT DL1 PN dendrites innervate the DL1 glomerulus specifically. Asterisks and dashed circles in $\boldsymbol{F}-\boldsymbol{K}, \boldsymbol{M}-\boldsymbol{N}$ denote cell bodies of single cell clones and the DL1 glomerulus innervated by these PNs, respectively. $\mathbf{G}-\boldsymbol{K}$, representative images of velo ${ }^{L 05209}$ mutant single neurons arranged into five classes according to their dendrite phenotypes. Class 1 velo ${ }^{L L 5209}$ mutant single neurons innervate the DL1 glomerulus often more sparsely and additionally innervate adjacent glomeruli partially, most often DL5 and D (G), whereas class 2 dendrites target dorsolateral glomeruli partially but avoid DL1 $(\boldsymbol{H})$. Class 3 velo ${ }^{\text {LL05209 }}$ mutant dendrites exhibit ventromedial innervation patterns, most typically mistargeting to VM6 $(I)$, class 4 dendrites innervate structures outside the AL, mostly the suboesophagial ganglion (SOG; arrowhead in $J$ ), and class 5 dendrites target nonstereotypically to multiple glomeruli within the AL ( $\boldsymbol{K}$, arrowheads). $\boldsymbol{L}$, Quantification of the five distinct dendrite mistargeting classes is shown in percentages ( $y$-axis) for three velo alleles ( $X$-axis). Numbers in brackets represent phenotypic class. Numbers on top of the bars indicate the number of velo mutant DL1 single neurons analyzed. $M, N$, During development at $50 \mathrm{~h}$ APF (after pupa formation) WT DL1 PN dendrites innervate the DL1 glomerulus $(\boldsymbol{M})$ while dendrites of a velo ${ }^{e 01260}$ mutant DL1 PN mistarget to the ventromedial area $(\boldsymbol{N})$. All images are confocal z-projections, mCD8-GFP labeled dendrites and cell bodies generated by MARCM using Gal4-GH146 are marked in green, the presynaptic marker nc82 is shown in red. Scale bars: $20 \mu \mathrm{m}$. Genotype: yw hsFIp122 UAS-mCD8GFP; Gal4-GH146 UAS-mCD8GFP/+; Gal80 FRT2A/ FRT2A, FRT82B, y+ (B, D, F, M), yw hsFlp ${ }^{122}$ UAS-mCD8GFP; Gal4-GH146 UAS-mCD8GFP/+; Gal80 FRT2A/ velo $^{\text {LLO5209 }}\left[\right.$ DsRed] FRT2A, FRT82B, $y+(\boldsymbol{C}, \boldsymbol{E}, \mathbf{G}-\boldsymbol{K})$, and yw hsFIp ${ }^{122}$ UAS-mCD8GFP; Gal4-GH146 UAS-mCD8GFP/ +; Gal80 FRT2A/velo ${ }^{\text {e01260 }[W+] ~ F R T 2 A, ~ F R T 82 B ~(N) . ~}$

to label PNs from two neuroblast lineages, anterodorsal $(\mathrm{ad})$ and lateral (1) PNs (Jefferis et al., 2001). Wild-type (WT) adPNs and lPNs targeted stereotyped sets of glomeruli in neuroblast clones (Fig. $1 B, D)$. PNs of $C G 10107$ mutant neuroblast clones exhibited several defects (Fig. 1C,E). First, the number of neurons was reduced from an average of 35 adPNs in WT (Jefferis et al., 2001) to 5 neurons in velo mutant clones (quantified in Fig. 4G). Second, the overall dendritic mass was reduced and disorganized. Third, dendrites innervated incorrect glomeruli within the antennal lobe (AL; Fig. $1 C$ ), or ectopically projected outside the AL (Fig. $1 E$ ). Because dendrites lost their path in CG10107 mutant neurons, we named this gene verloren (velo), meaning "lost" in German. 
To study dendrite targeting in greater detail, we used Gal4-GH146 and MARCM to label single cell PN clones produced in newly hatched larva. In WT, they always projected their dendrites to the posterior, dorsolateral glomerulus DL1 in the AL, hereafter referred to as DL1 PN (Fig. 1 F; Jefferis et al., 2001). This specific innervation pattern was formed during early pupa development (Fig. 1M; Jefferis et al., 2004). In velo mutant DL1 PNs, we observed various dendrite mistargeting defects that were already manifested during pupa development (Fig. $1 N$ ). In adults, these phenotypes could be grouped into five distinct phenotypic classes. In class 1 , dendrites innervated DL1 sparsely but spilled over into incorrect adjacent areas (Fig. $1 G$ ). In class 2 , dendrites were found in the dorsolateral region of the AL but excluded the DL1 glomerulus (Fig. $1 \mathrm{H})$. Class 3 mutant DL1 PNs projected to ventromedial regions in the $\mathrm{AL}$, preferably but not exclusively to the VM6 or VC3 glomeruli (Fig. 1I). Class 4 mutant DL1 PNs mistargeted their dendrites outside the AL mostly into the subesophageal ganglion (Fig. $1 \mathrm{~J}$ ). Class 5 mutant dendrites innervated multiple glomeruli within the AL (Fig. $1 K)$. The severity of dendrite mistargeting phenotypes increased from class 1 to 5 , with class 1 being the mildest and closest to the DL1 glomerulus. We quantified the penetrance of the different phenotypic classes for these 3 velo alleles. We determined that e01260 was the strongest allele, lacking PNs of class 1 that targeted to or close to DL1. LL05207 was the weakest allele lacking class 4 and 5 phenotypes, and LL05209 represented the intermediate allele with all 5 phenotypic classes present (Fig. $1 L$ ). The severity of the phenotypes across different alleles, as measured by DL1 PN mistargeting, and the presence of mistargeting phenotypes in all alleles led us to conclude that velo is required for $\mathrm{PN}$ target selection. This was supported by further evidence below.

\section{velo is required for $\mathrm{PN}$ axon morphogenesis}

In addition to dendrite mistargeting, we also observed defects in axon morphologies of velo mutant DL1 PNs. WT DL1 PN axons project stereotypically into the lateral horn ( $\mathrm{LH})$ after passing through the mushroom body calyx (MBC), where they form $\sim 5$ collateral branches. After entering the LH, DL1 PN axons always form one characteristic dorsal branch (arrowhead in Fig. 2B) while the main branch terminates at the lateral edge of the $\mathrm{LH}$ (Fig. 2 B; Marin et al., 2002; Wong et al., 2002; Jefferis et al., 2007). In velo mutant DL1 PNs, axons extended along the normal path and always reached the end of the $\mathrm{LH}$, but formed only $0-2$ collaterals in the $\mathrm{MBC}$, and had often a missing or shorter dorsal branch in the LH (Fig. 2D,F,G). These axonal phenotypes were independent of the phenotypic class of dendrite mistargeting described earlier (Fig. 2D,F) and occurred in multiple velo alleles, including LL05209 (Fig. 2D,F) and e01260 (Fig. $2 H$ ). However, dendrite


Figure 2. Velo is required for stereotyped PN axon pattern formation. $\boldsymbol{A}-\boldsymbol{H}, \mathrm{PN}$ dendrites $(\boldsymbol{A}, \boldsymbol{C}, \boldsymbol{E}, \boldsymbol{G})$ and axons $(\boldsymbol{B}, \boldsymbol{D}, \boldsymbol{F}, \boldsymbol{H})$ of single neurons generated by MARCM are shown in green stained with antibodies to $C D 8$, red represents staining using the are within the dotted lines, an arrowhead points to the dorsal branch $(\boldsymbol{B})$. $\boldsymbol{C}-\boldsymbol{F}$, Representative images for two 作 $\mathrm{MBC}$ and lack the dorsal branch in the $\mathrm{LH}(\boldsymbol{D}, \boldsymbol{F}) . \mathbf{G}, \boldsymbol{H}$, Typical velo ${ }^{\text {e01260 }}$ single PNs mistarget ventromedially, often to the VM6 glomerulus $(\boldsymbol{G})$ and their corresponding axon bears fewer branches in the $\mathrm{MBC}$ and $\mathrm{LH}$ or lacks them entirely $(\boldsymbol{H})$. $\boldsymbol{A}-\boldsymbol{H}$ represent confocal z-projections. Scalebars: $20 \mu \mathrm{m}$. Genotypes:ywhsFlp ${ }^{122}$ UAS-mCD8GFP;Gal4-GH146UAS-mCD8GFP/+;Gal80FRT2A/FRT2A, FRT82B, y+ $(\boldsymbol{A}, \boldsymbol{B})$,ywhsFlp ${ }^{122}$ UAS-mCD8GFP;Gal4-GH146UAS-mCD8GFP/+;Gal80FRT2A/velo ${ }^{\text {LO05209 }}\left[\right.$ DsRed] FRT2A, FRT82B, y+ (C-F),ywhsFlp ${ }^{122}$ UAS-mCD8GFP; Gal4-GH146 UAS-mCD8GFPI + ; Gal80 FRT2A/velo ${ }^{e 01260}[W+]$ FRT2A, FRT82B $(\boldsymbol{G}, \boldsymbol{H})$.

class 3 mutant DL1 PNs (ventromedial mistargeting, particularly to the VM6 glomerulus) often exhibited extensive, but nonstereotyped axon branching within the $\mathrm{LH}$ (Fig. $2 \mathrm{E}-\mathrm{H}$ ).

\section{velo is cell-autonomously required in postmitotic neurons}

Transposon insertions can cause effects in genes distant to their insertion site. To determine that the loss of Velo function was indeed the cause for the mutant phenotypes, we used two approaches. First, we performed precise excision of two transposon insertions in velo, LL05207 and LL05209, which fully reverted PN dendrite and axon morphogenesis defects (data not shown). Second, we generated UAS-velo-HA rescue transgenes using the coding sequence of the long transcript (Fig. 1A). MARCM overexpression of Velo-HA in WT DL1 single PNs did not result in detectable phenotypes in their dendrites and axons (data not shown), but rescued the velo mutant dendrite and 
$e 01260+$ UAS-veloHA
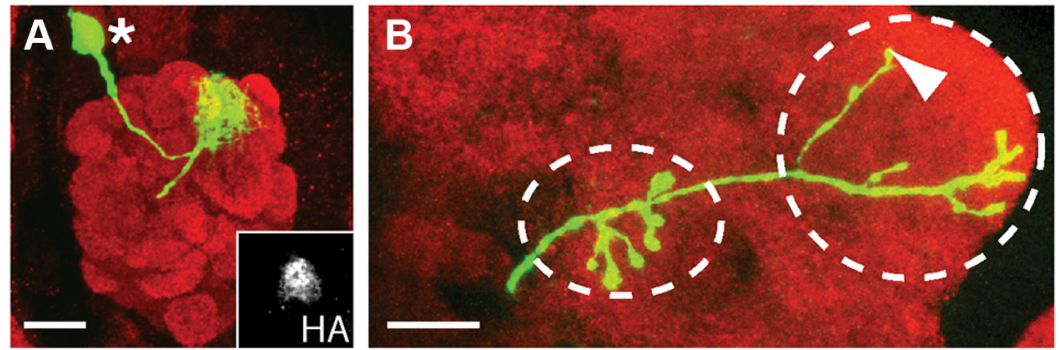

\section{C $\square>3$ branches 0-2 branches}

$\mathrm{MBC}$ branches
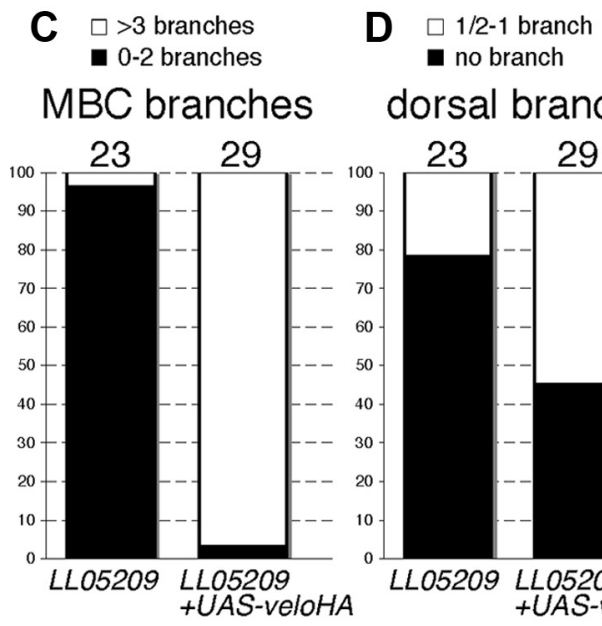

dorsal branch



$E$

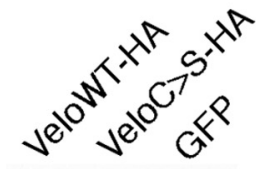

250-



$50-$



$\mathrm{HA}$

25-

$S_{H A}$
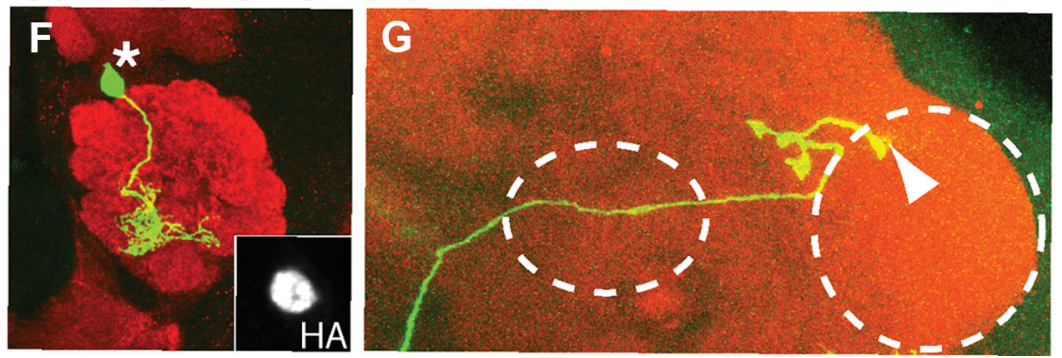

Figure 3. Velo functions in postmitotic PNs and requires a catalytically intact protease to regulate dendrite and axon targeting. $A-B$, Postmitotic expression of UAS-velo-HA in single DL1 velo mutant PNs with Gal4-GH146 results in localization of the Velo-HA fusion protein to the nucleus $\left(\boldsymbol{A}\right.$, inset), and rescues velo ${ }^{e 01260}$ mutant dendrite phenotypes in 20/22 cases $(\boldsymbol{A})$ and axon $(\boldsymbol{B})$ phenotypes as quantified. $C-D$, Quantification of the PN axon morphogenesis phenotypes in the $\mathrm{MBC}(\boldsymbol{C})$ and the dorsal branch in the $L H(D)$ are illustrated as percentages of phenotypes on the $y$-axis and genotypes on the $x$-axis. C, Single DL1 PNs are divided into groups that contain $0-2$ or $>3$ branches in the MBC. $D$, Additionally, single PNs are examined for the presence of a dorsal branch in the LH. Numbers on top of the bars indicate the number of single PNs analyzed. E, VeloWT-HA, VeloC $>$ S-HA or GFP were expressed in $\mathrm{S} 2$ cells and detected by Western blot. Expression levels were similar for both WT and mutant proteins. $\alpha$-Tubulin was used as a loading control. $\boldsymbol{F}, \mathbf{G}$, MARCM experiments expressing a catalytically dead Velo protease UAS-velo ${ }^{\mathrm{C}}$ - HA in velo ${ }^{\text {e01260 }}$ mutant PNs fail to rescue $P N$ dendrite in $8 / 8(\boldsymbol{F})$ and axon $(\boldsymbol{G})$ phenotypes in $5 / 8$ cases, and even causes premature axon termination outside the LH in $3 / 8$ ( $\boldsymbol{G}$, arrowhead). $\boldsymbol{A}, \boldsymbol{B}, \boldsymbol{F}, \boldsymbol{G}$, Confocal $z$-projections stained with anti-CD8 (green), nc82 (red), and anti-HA ( $\boldsymbol{A}$, $\boldsymbol{F}$, insets), except insets in $\boldsymbol{A}, \boldsymbol{F}$ are single confocal sections. Asterisks in $\boldsymbol{A}, \boldsymbol{F}$ mark single cell bodies. Scale bars: $20 \mu \mathrm{m}$. Genotypes: ywhsFlp 122 UAS-mCD8GFP; Gal4-GH146 UAS-mCD8GFP/UAS-velo-HA ${ }^{\# 108 L}$; Gal80 FRT2A/velo ${ }^{\text {e01260 [ }}$ + ] FRT2A, FRT82B $(\boldsymbol{A}, \boldsymbol{B})$, yw hsFIp ${ }^{122}$ UAS-mCD8GFP; Gal4-GH146UAS-mCD8GFP/ + ; Gal80 FRT2A/velo ${ }^{\text {e01260 }}[\mathrm{W}+]$, FRT2A, FRT82B UAS-velo ${ }^{\odot S}-\mathrm{HA}^{\# 1.2}(\boldsymbol{F}, \boldsymbol{G})$.

axon phenotypes (Fig. $3 A, B)$. Twenty of $22(91 \%)$ examined velo mutant DL1 PNs normally innervated the DL1 glomerulus when one copy of UAS-velo-HA was simultaneously expressed in this neuron (compare Figs. $2 C, E, G, 3 A$ ).

To examine the axon morphogenesis defects, we quantified the velo mutant axon phenotypes by counting the number of collateral branches in the MBC (Fig. 3C) and detecting the dorsal branch in the LH (Fig. 3D). In $96 \%$ of velo mutant DL1 axons 2 or fewer collaterals innervated the MBC. Upon introduction of a velo rescue transgene, only $3 \%$ of examined DL1 axons had 2 or fewer collaterals in the
MBC whereas $97 \%$ had 3 or more collaterals (Fig. 3C). We observed similar rescue effects for the dorsal branch of DL1 axons: in 78\% of velo mutant axons the dorsal branch was missing, a phenotype reverted partially upon rescue with the velo transgene (Fig. $3 D)$. Thus, we conclude that velo is required cell-autonomously for stereotyped dendrite innervation and axon branch formation of DL1 PNs. Moreover, because Gal4-GH146 expression is restricted to postmitotic neurons (Spletter et al., 2007), these rescue experiments also demonstrated that velo regulates dendrite and axon morphogenesis in postmitotic neurons.

The catalytic domain of Velo is essential for its function in PNs

Sequence analysis reveals that velo encodes a SUMO protease. The catalytic domain was shown to be essential for the protease activity in human homologs of Velo (Lima and Reverter, 2008; Shen et al., 2009). To test whether Velo requires its catalytic domain for regulating neuronal targeting, we generated a rescue transgene bearing a mutation in the catalytic domain of the protease. We introduced a mutation C1624S that disrupts the core catalytic triad Cys-His-Asp predicted to yield a catalytically dead variant. We found that WT and mutant Velo proteins were present at similar levels when expressed in cell culture (Fig. 3E), indicating that the C1624S mutation did not cause destabilization of the Velo protein. We introduced one copy of the modified transgene, UAS-velo ${ }^{C>S}$-HA, into velo mutant DL1 PNs. We did not detect any rescue in dendrite mistargeting in $8 / 8$ cases (Fig. $3 F$ ). This was not due to low or absent expression of the transgene, as we can readily detect high levels of the epitope-tagged Velo ${ }^{\mathrm{C}>\mathrm{S}}$-HA in the nucleus (Fig. 3F, inset). The nuclear localization was consistent with the expression of WT UAS-velo-HA transgene (Fig. $3 A$, inset) and suggests that Velo likely acts predominantly in the nucleus. In addition to the failure to rescue DL1 dendrite mistargeting, expression of $\mathrm{Velo}^{C>S}$-HA in velo mutant DL1 PNs also did not rescue the axon phenotypes. 5/8 DL1 PN axons still exhibited the same axon morphology defects regarding $\mathrm{MBC}$ branching and dorsal branch formation; furthermore, 3/8 DL1 PN axons failed to innervate the LH (Fig. $3 G$ ). In summary, we conclude that the catalytic domain of Velo is essential for its role during dendrite target selection and axon morphogenesis in PNs. Therefore, Velo indeed might act as SUMO protease, likely in the nucleus.

Two other SUMO proteases can partially compensate for Velo's functions in PNs

Velo is highly conserved across species from yeast to human. Sentrin-specific protease 7 is the closest human homolog (Fig. 
$4 A$ ). Velo belongs to the family of C48 cysteine proteases responsible for the processing and deconjugation of SUMO proteins. SUMO proteases are abundant in eukaryotes including two members in budding yeast, the Ubiquitin-like proteinspecific proteases (Ulp), and seven in mammals, called Sentrin-specific Proteases (SENPs). SUMO proteases diverge into two major branches, one related to Ulp1 and the other Ulp2 (Hay, 2007; Mukhopadhyay and Dasso, 2007). Velo has a split C48 catalytic domain characteristic of SENP6 and SENP7. Our phylogenetic analysis of sequence similarities classified it as Ulp2 related protease (Fig. 4A).

Protein sequence comparisons predict five SUMO proteases in the Drosophila genome: three are Ulp1-related (DmUlp1, CG11023 and CG32110) and two are more similar to Ulp2 (velo, CG12717) related. Before this study, none had been studied in vivo, nor were reagents for mutant and overexpression analysis available. To assess whether Velo is uniquely required in PN targeting, we generated mutants for the closest velo ortholog, CG12717, using FLP recombinase-mediated excision (Parks et al., 2004). The flanking P-element $P(X P) d 05069$ and piggyBac line $p B A C(R B) e 01706 a$ allowed us to delete the entire open reading frame of CG12717, while not interfering with adjacent genes (Fig. $4 B$ ). We recombined the resultant LOF mutant CG12717 $7^{\text {ex\#6 }}$ with FRT19A, performed MARCM experiments, and found that dendrites and axons targeted normally in CG12717 $7^{\text {ex\#6 }}$ neurons (Fig. $4 C$ and data not shown). PN cell numbers were normal in neuroblast clones (Fig. 4D), and CG12717 mutant single DL1 PNs normally innervated the DL1 glomerulus (Fig. 4C, compare to Fig. $1 F$ ). Therefore, we conclude that CG12717 is either not required or acts redundantly with Velo during PN target selection.

To address the question of whether CG12717 or another SUMO protease from the Ulp1 family, DmUlp1, can functionally compensate for Velo in PN targeting, we generated HA-tagged transgenes for these two SUMO proteases and expressed them in velo mutant PNs. We observed a significant reversion of the DL1 mistargeting phenotype in velo mutant PNs supplemented with DmUlp1-HA; 12 of 14 (86\%) examined PN dendrites target to the DL1 glomerulus (Fig. 4F). Similar experiments using UAS-CG12717-HA also yielded a significant rescue in DL1 targeting, although to a lesser extent (5 of 13 innervate DL1; data not shown). However, velo mutant axon phenotypes could not be reverted by expressing either DmUlp1-HA or CG12717-HA in DL1 PNs (data not shown). This suggests that other SUMO proteases of both families can
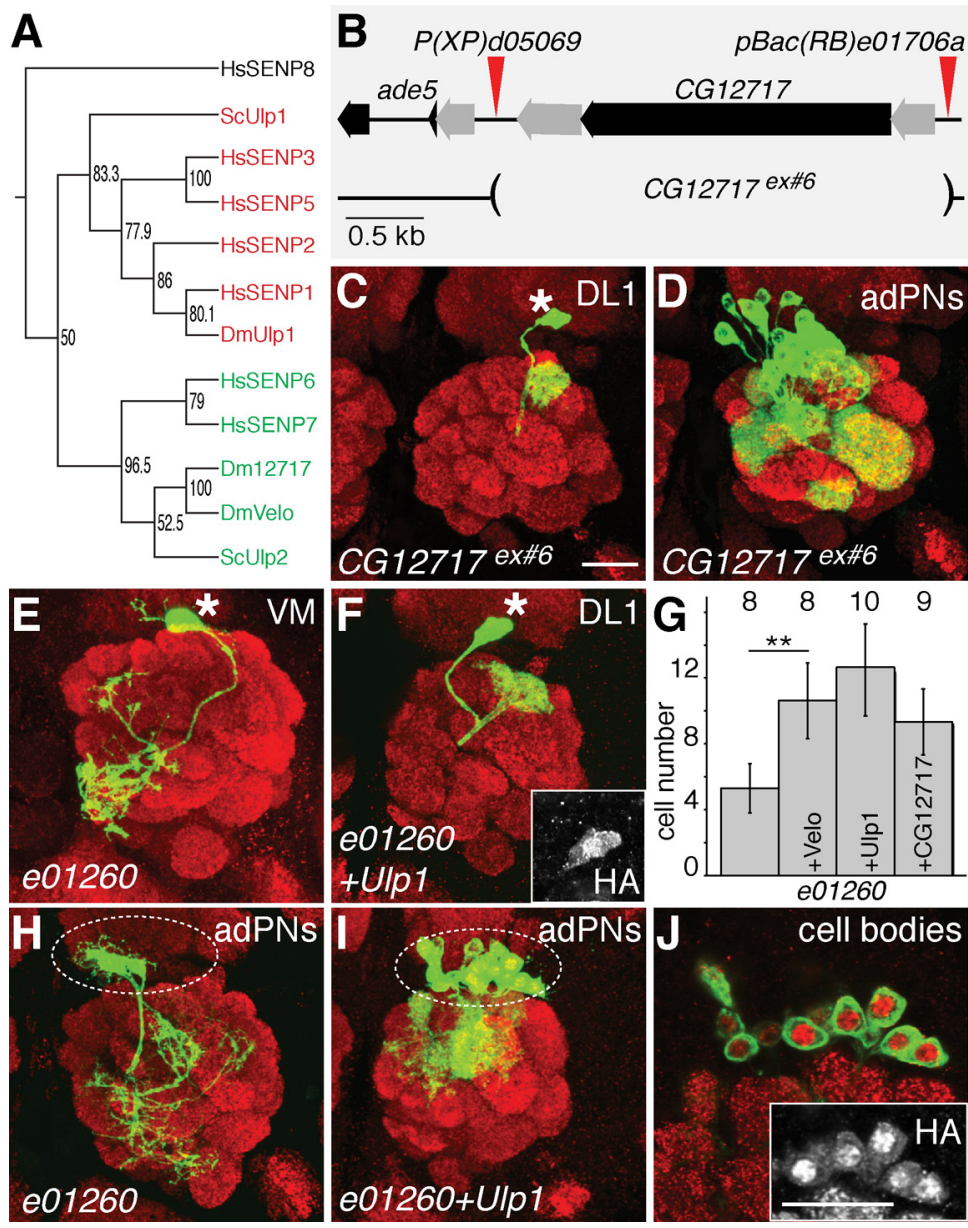

Figure 4. Other SUMO proteases can complement Velo's function in PN target selection and neuronal survival. $\boldsymbol{A}$, Phylogenetic tree displaying the relationship between budding yeast $(\mathrm{Sc})$, human $(\mathrm{Hs})$, and Drosophila $(\mathrm{Dm})$ homologs of SUM0 proteases. The number at each branch represents the confidence in that branch, as generated by bootstrapping analysis. For details about the making of this tree, see Materials and Methods, Generation of phylogenetic tree. The ScUlp1-related family is illustrated in red, ScUlp2-related members in green letters. $\boldsymbol{B}$, Schematic of the CG12717 gene locus indicating the insertions of the P-element $P(X P) d 05069$ and pBac(RB)e01706a lines (Thibault et al., 2004; red triangles) used to generate the deletion mutant CG12717 $7^{\text {ex\#6 }}$ by FLP-mediated excision. Brackets illustrate the extent of deletion. Exons are shown as black, UTRs as gray bars, and introns as lines. C, D, CG12717 $7^{\text {ex\#6 }}$ adPNs and single DL1 PNs target, like WT PNs, target a stereotyped set of glomeruli and to the DL1 glomerulus, respectively. $\boldsymbol{E}, \boldsymbol{F}$, Typical velo ${ }^{\text {e01260 }}$ single PNs mistarget ventromedially (VM), often to the VM6 glomerulus (E) which is rescued by adding UAS-DmUlp1-HA into the same mutant neuron in 12/14 PNs $(\boldsymbol{F})$. Inset in $\boldsymbol{F}$ demonstrates subcellular distribution of the DmUlp1 fusion protein. Asterisks in $\boldsymbol{C}, \boldsymbol{E}, \boldsymbol{F}$ denote single cell bodies. $\boldsymbol{H}-\boldsymbol{J}$, velo ${ }^{e 01260}$ adPN cell numbers are reduced $(\boldsymbol{H})$, a phenotype that is significantly rescued by introducing a UAS-DmUIp1-HA transgene $(\boldsymbol{I})$. $\boldsymbol{H}, \boldsymbol{I}$, adPNs are encircled by dotted lines. $\boldsymbol{J}$, DmUlp1-HA (in red and inset) localizes to the nucleus and cytoplasm. G, Quantification of adPN numbers in velo ${ }^{\text {e01260 }}$ neuroblast clones and after introducing various UAS rescue transgenes. Numbers on top of the bars indicate the number of analyzed anterodorsal neuroblast clones. Error bars indicate SEM. ${ }^{* *} p<0.00002$ in two-tailed $t$ test. $\boldsymbol{C}-\boldsymbol{F}, \boldsymbol{H}-\boldsymbol{I}$, confocal z-projections stained with anti-CD8 (green), nc82 (red), and anti-HA ( $\boldsymbol{F}, \boldsymbol{J}$, insets), except $\boldsymbol{J}$ and insets in $\boldsymbol{J}, \boldsymbol{F}$ are single confocal sections. Scale bars: 20

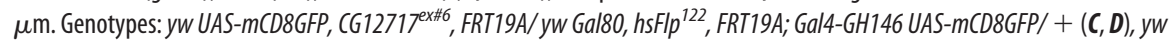
hsFlp ${ }^{122}$ UAS-mCD8GFP; Gal4-GH146 UAS-mCD8GFP/ +; Gal80 FRT2A/ velo ${ }^{e 01260}[w+]$, FRT2A, FRT82B (E, H, J), yw hsFlp ${ }^{122}$ UAS-mCD8GFP; Gal4-GH146 UAS-mCD8GFP/ UAS-DmUIp1-HA ${ }^{\# 5.1}$; Gal80 FRT2A/velo ${ }^{e 01260}[\mathrm{~W}+]$, FRT2A, FRT82B $(\boldsymbol{F}, \mathbf{G}, \mathbf{I}, \boldsymbol{J}), \mathrm{yw}$ hsFlp ${ }^{122}$ UAS-mCD8GFP; Gal4-GH146 UAS-mCD8GFP/ UAS-velo-HA ${ }^{\# 108 L}$; Gal80 FRT2A/ velo ${ }^{e 01260}[w+]$, FRT2A, FRT82B and $y w$ hsFIp ${ }^{122}$ UAS-mCD8GFP; Gal4-GH146 UAS-mCD8GFP/UAS-CG12717-HA ${ }^{\# 4}$; Gal80 FRT2A/velo ${ }^{\text {e01260 }}[\mathrm{W}+]$, FRT2A, FRT82B (G).

compensate for the function of Velo in PN dendrite targeting but not axon arborization.

Aberrant sumoylation can lead to many biological malfunctions, including defects during cell division (Di Bacco et al., 2006). In velo mutant neuroblast clones $P N$ cell numbers were drastically reduced from $\sim 35$ neurons in WT (Jefferis et al., 2001) to an average of 5.5 neurons per ad neuroblast clone (Figs. $1 C$, $4 G, H)$. By driving UAS-Velo-HA expression in velo mutant ad PNs, this defect was partially rescued as the number of neurons 


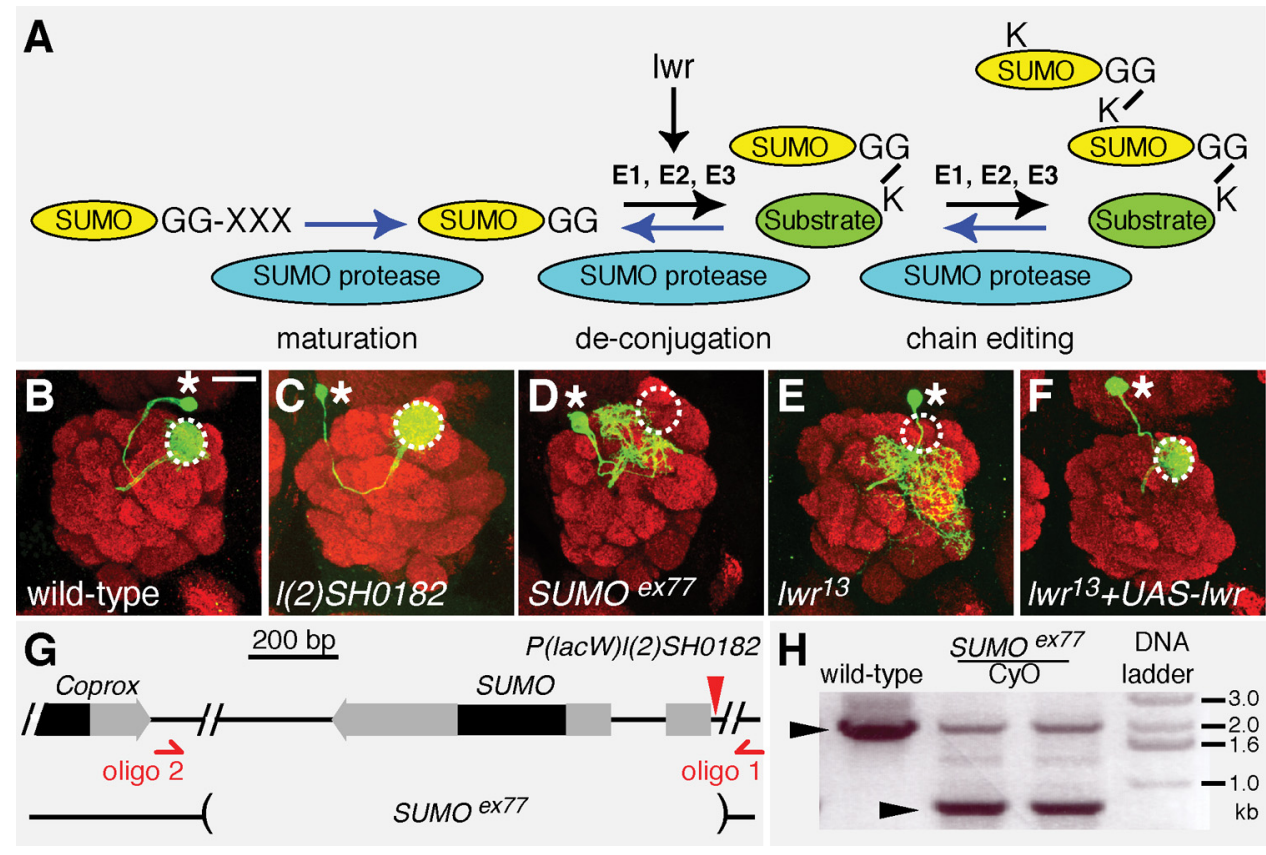

Figure 5. SUMO and an E2 conjugating enzyme are also required for PN dendrite targeting. $A$, Schematic representation of reactions catalyzed by SUMO proteases in the protein sumoylation pathway. The processing reaction requires a SUMO protease (blue) and involves cleavage of a peptide bond within the SUMO (yellow) propeptide to expose the C-terminal di-glycine motif. E1, E2, and E3 are the activating enzyme, conjugating enzyme and SUMO ligase, respectively, and are required for SUMO conjugation (black arrow). The lesswright (Iwr) gene encodes the only E2 enzyme in Drosophila. SUMO deconjugation requires cleavage of the amide bond between mature SUMO and the target lysine within the substrate (green). Chain editing is chemically identical to deconjugation, although it is distinguished by cleavage of one or more SUMOs from a poly-SUMO chain. SUMO catalyzed reactions are indicated by blue arrows. $\boldsymbol{B}$, WT DL1 PN dendrites innervate the DL1 glomerulus specifically. C, D, PNs mutant for the original P-element /(2)SH0182 (G) exhibit normal DL1 innervation (C), while SUM0 ${ }^{\text {ex77 }}$ DL1 PN dendrites fail to innervate the DL1 glomerulus (D). $\boldsymbol{E}, \boldsymbol{F}, \mathrm{Iwr}^{13} \mathrm{DL} 1 \mathrm{PNs}$ mistarget their dendrites ventromedially from DL1, avoiding DL1 $(\boldsymbol{E})$; this phenotype is fully rescued by introducing a UAS-Iwr transgene in $12 / 12$ single neurons $(\boldsymbol{F})$. $\boldsymbol{B}-\boldsymbol{F}$, Confocal z-projections stained with nc82 as a presynaptic marker (red) and anti-CD8 (green) for PN single cell bodies (asterisks) and their dendrites. Dashed circles outline the DL1 glomerulus. Scale bar: 20 $\mu \mathrm{m}$. G, Schematic of the SUMO gene locus showing the P-element insertion /(2)SH0182 (red triangle) 10 bp from the transcriptional start. I(2)SH0182 was used for the imprecise excision to generate the SUMO ${ }^{\text {ex77 }}$ deletion allele (shown by brackets). The positions of primers to screen for imprecise excision mutations are denoted with red arrows. Exons are shown as black, UTRs as gray bars, and introns as lines. $\boldsymbol{H}, 1 \%$ Agarose gel showing a $\sim 2000$ bp DNA PCR product amplified with the primers shown in $\boldsymbol{G}$ from WT fly genomic DNA (lane 1). From SUMO ${ }^{e x 77}$ heterozygous fly genomic DNA two fragments are amplified denoted by black arrowheads (lane $2+3): 2000$ bp for the wt and 800 bp for the deletion chromosome, indicating a $\sim 1200$ bp deletion in SUMO ${ }^{\text {ex77 }}$ mutants. Standard DNA ladder is loaded in right lane. Genotypes: yw hsFIp ${ }^{122}$ UAS-mCD8GFP; FRT40A Gal4-GH146 UAS-mCD8GFP/ FRT40A (B), yw hsFIp ${ }^{122}$ UAS-mCD8GFP; FRT40A Gal4-GH146 UAS-mCD8GFP/

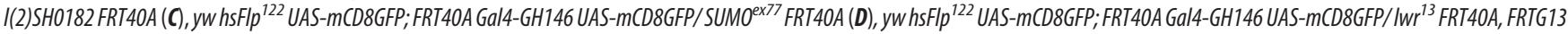
(E), yw hsFlp ${ }^{122}$ UAS-mCD8GFP/UAS-Iwr; FRT4OA Gal4-GH146 UAS-mCD8GFP/Iwr ${ }^{13}$ FRT40A, FRTG13 (F), $w^{1118}$ and $y w ;$ SUMO $0^{\text {ex77 } F R T 40 A / C y O ~}(\boldsymbol{H})$.

was doubled ( $\sim 10.6$ neurons; Fig. $4 G)$. The number of adPNs also increased upon expressing UAS-DmUlp1-HA $(\sim 12.5$ neurons) or UAS-CG12717-HA ( $\sim 9.3$ neurons) in velo mutant neuroblast clones (Fig. 4G,I). The slight variability of the rescued cell numbers could reflect different expression levels of the distinct transgenes. The fact that cell numbers can be rescued partially via the postmitotic expression of various SUMO proteases in velo mutant ad PNs suggest that velo is required for neuronal survival. However, the fact that cell numbers in the rescued adPNs were markedly less than WT suggests that Velo also functions during neuroblast proliferation.

We further noted a difference in the subcellular distribution of the three SUMO protease transgenes. While Velo-HA was found exclusively in the nucleus (Fig. 3A, inset), DmUlp1-HA and CG12717-HA localized both to the nucleus and cytoplasm, although neither could be detected in dendrites or axons (Fig. 4J, inset in $4 F$, and data not shown). This difference in subcellular distribution may indicate differential neuronal functions for each SUMO protease. Based on these expression experiments, the three proteases appeared to be equally potent in the partial rescue of cell numbers (Fig. 4G). Moreover, DmUlp1-HA and CG12717-HA (to a lesser extent) can functionally substitute for Velo in PN dendrite targeting. However, axonal morphogenesis appears to specifically require Velo.

\section{SUMO and an E2 conjugating enzyme are required for PN dendrite targeting}

Sumoylation is a reversible post-translational modification and many enzymes participate in SUMO conjugation and deconjugation (Johnson, 2004; Geiss-Friedlander and Melchior, 2007). SUMO proteases cleave SUMO into its mature form to expose the C-terminal di-glycine motif that is subsequently conjugated to a lysine residue on the target substrate. This process requires an E1 activating enzyme, an E2 conjugating enzyme, and an E3 ligase. SUMO proteases deconjugate SUMO(s) from the substrate or from SUMO chains (chain editing; Fig. 5A).

The involvement of a SUMO protease in PN target selection prompted us to examine other components of the SUMO pathway in the same process. To this end, we tested mutants for SUMO and the E2 conjugating enzyme lesswright (lwr) for their role in dendrite target selection in DL1 PNs using MARCM. We identified a P-element, $P(\operatorname{lac} W) l(2) S H 0182[w+]$, which is inserted in the promoter region of the SUMO gene (Fig. 5G, red triangle). PN targeting to the DL1 glomerulus was normal when DL1 PNs $(n=10)$ were homozygous for this insertion (compare Fig. $5 B, C$ ). As this insertion might not impair SUMO function, we generated SUMO mutants via imprecise excision of $P(l a c W) l(2) S H 0182[w+]$ by screening for white eyes and mapping the extent of the deletion by PCR. We identified a $\sim 1200 \mathrm{bp}$ 
deletion, SUMO ${ }^{\text {ex77 }}$, that covered the entire SUMO gene but left neighboring genes unaffected (Fig. 5G,H). After recombining the $S U M O^{e x 77}$-null allele onto FRT40A, we performed MARCM experiments. This yielded a very low clonal frequency and often resulted in lethality in both larvae and pupae, suggesting that SUMO is required for many essential functions in cells and even the generation of homozygous mutant clones cannot prevent animal death. However, from the four single cell clones we obtained from $\sim 500$ dissected brains, we found dendrite mistargeting in each case (Fig. 5D; data not shown). As such, we conclude that SUMO is essential for PN target selection.

Next, we examined the function of $l w r$ in PN target selection by inducing MARCM clones of the LOF allele $l w r^{13}$ (Sun et al., 2003). Dendrites of $l w r^{13}$ mutant DL1 PN single cell clones innervated large areas across the entire AL. In 14 of 24 (58\%) cases, lw $r^{13}$ DL1 PN dendrites shifted ventromedially from DL1 and avoided the DL1 glomerulus (Fig. 5E); 5/24 (21\%) innervated the DL1 glomerulus but spill into several adjacent glomeruli, and the remaining 5/24 (21\%) innervate simultaneously dorsolateral and ventromedial portions of the AL (data not shown). All dendrite mistargeting phenotypes were fully rescued by supplementing single $l w r^{13}$ PNs with UAS-lwr (12/ 12, Fig. $5 F$ ).

We conclude from our data examining the two LOF alleles, SUMO ${ }^{\text {ex77 }}$ and $l w r^{13}$, that sumoylation in PNs is cellautonomously required for the correct target selection of their dendrites within the AL. The rescue experiment for $l w r$ further indicated that this E2 SUMO conjugating enzyme acts in postmitotic neurons to regulate PN dendrite targeting. The neuronal targeting defects of two members of the sumoylation pathway and Velo support the notion that the function of Velo in $\mathrm{PN}$ dendrite targeting is related to its role in regulating sumoylation.

\section{Genetic evidence that Velo acts in SUMO deconjugation rather than in SUMO maturation}

SUMO proteases can act at two distinct steps in the sumoylation pathway. First, they promote sumoylation by cleaving immature SUMO into its active form. Second, they revert sumoylation by cleaving off one or more SUMOs from their substrates (Fig. 5A).

We performed genetic interaction experiments to distinguish whether Velo promotes (Fig. 6A, model 1) or reverts (Fig. 6A, model 2) sumoylation. We took advantage of the fact that the velo ${ }^{L 05209}$ allele produced an intermediate dendrite mistargeting phenotype in DL1 PNs that might be sensitive to both enhancement and suppression of the phenotype (Figs. $1 \mathrm{~L}, 6 \mathrm{E}$ ). A typical example (38\%) for a velo ${ }^{L 205209}$ DL1 PN exhibiting ventromedially mistargeted dendrites is presented in Figure $6 \mathrm{~B}$. We generated velo ${ }^{L 05209}$ DL1 PNs using MARCM and reduced SUMO or Lwr protein levels by half using flies heterozygous for the $S U M O^{\text {ex77 }}$ or $l w r^{13}$ LOF alleles, respectively. If Velo functions in
SUMO maturation, we would expect an enhancement of the velo $^{L 205209}$ phenotype leading to a decrease in neurons causing the mild class 1 phenotype (Fig. $1 G$ ). However, if velo acts in SUMO deconjugation, we would expect a suppression of the dendrite mistargeting phenotype causing an increase in neurons exhibiting the mild class 1 phenotype.

We observed suppression of dendrite mistargeting in velo $^{\text {LL05209 }}$ mutant DL1 PNs with either SUMO or Lwr protein levels reduced by half in the same mutant neuron (Fig. 6C-E). Quantifications of the various phenotypic classes representing dendrite targeting defects for single PNs of the three distinct genotypes (Fig. 6E) suggested that Velo acts predominantly in SUMO deconjugation rather than in SUMO maturation. Our finding is consistent with previous results that the closest human homolog of Velo, SENP7, is unable to process SUMO precursors for SUMO maturation but rather contributes to SUMO deconjugation from poly-SUMO chains (Shen et al., 2009).

\section{Discussion}

Protein sumoylation plays an important role in a wide range of cellular processes, including transcription, chromosome organization and function, DNA repair, nuclear transport, signal transduction, and cell cycle progression. Since its discovery, several 
hundred sumoylation substrates have been identified, including proteins localized to the nucleus, cytoplasm, or at the plasma membrane (Johnson, 2004; Wilkinson and Henley, 2010). Recent studies have shown that many of these sumoylated substrates are crucial for neuronal development and function (Wilkinson et al., 2010). However, because the major components of the sumoylation pathway are essential for cell viability, it is challenging to examine the specialized functions of these enzymes and hence the effects of sumoylation in vivo.

Here, from a forward genetic screen using a powerful mosaic analysis technique, we identified a predicted SUMO protease, Velo, that regulates dendrite and axon targeting in postmitotic neurons in vivo. Several lines of evidence indicate that Velo controls neuronal morphogenesis by regulating protein sumoylation. First, the catalytic domain of the protease is required for its function in neurons. Second, the dendrite targeting phenotypes can partially be rescued by two other predicted SUMO proteases from two evolutionarily separable branches. SUMO proteases from the Ulp 1 family predominantly function in SUMO maturation and deconjugation of SUMO from mono-sumoylated substrates, while Ulp2-like proteases deconjugate SUMO protein(s) from poly-SUMO chains. Interestingly, overexpression of both Ulp1 and Ulp2 family proteases was able to rescue the velo dendrite phenotypes. Third, two other components of the sumoylation pathway, SUMO itself and the unique E2 conjugating enzyme Lesswright (Lwr), are also required cell-autonomously for PN dendrite targeting. Fourth, SUMO and Lwr exhibit dosage-sensitive interactions with Velo; velo mutant dendrite phenotypes were suppressed by reducing SUMO or lwr gene dosage by half. Indeed, the nature of these genetic interactions suggests that Velo acts primarily to reverse sumoylation via SUMO deconjugation rather than to promote sumoylation via SUMO maturation.

It has previously been shown that the knockdown of the Drosophila SUMO protease Ulp1 and overexpression of human SENP7 result in a change of total SUMO conjugates in cultured cells (Smith et al., 2004; Shen et al., 2009). We performed similar experiments to test the biochemical activity of Velo as a SUMO protease by overexpressing Velo in cultured cells. We have not been able to detect significant changes in the overall spectrum of SUMO conjugates upon Velo overexpression (data not shown). It is possible that Velo activity requires a cofactor that is absent in cultured cells, or that Velo's substrates are absent in cultured cells. For these reasons and other technical hurdles, such as the lack of a Velo-specific antibody and difficulty to express the large Velo protein in bacteria, biochemical evidence for Velo acting as a SUMO protease is still missing. We also cannot rule out the possibility that some of the effects of Velo on PN dendrite and axon targeting are caused by its action on substrates unrelated to the SUMO pathway.

Although velo, SUMO and lwr mutant PNs exhibit aberrant dendrite targeting, their phenotypes are not identical. One possibility for the phenotypic differences could be due to the redundant action of SUMO proteases either between members of the same branch or even the two distinct branches. For example, CG12717, the closest homolog of Velo, or the Ulp1-related DmUlp1 could act redundantly with Velo during PN target selection. This is consistent with the fact that the overexpression of transgenes for both proteases can partially revert velo mutant dendrite phenotypes. However, the Drosophila genome only contains one gene encoding for SUMO and one for an E2 conjugating enzyme. Therefore, their loss-of-function phenotypes are more severe. Another possibility for the phenotypic differences we ob- serve in velo, SUMO and lwr mutant PNs could be attributed to the differential perdurance of these proteins in single neurons generated by MARCM. Finally, the two members of the sumoylation pathway we examined act in opposite ways with Velo: SUMO and Lwr promote, whereas Velo reverts, sumoylation. This feature implies that the dynamics of sumoylation are essential for dendrite and axon targeting: too much or not enough sumoylation are both harmful to PNs and cause neuronal mistargeting. Although all three possibilities can contribute, the last one might contribute most to the observed phenotypic differences.

The closest human homolog to Velo is SENP7 (Fig. 4A). SENP7 localizes to the nucleoplasm, consistent with our findings regarding Velo protein distribution. The catalytic domain of SENP7 is essential for its protease activity. Biochemical assays revealed that this protease functions preferably during deconjugation of poly-SUMO chains (Lima and Reverter, 2008; Shen et al., 2009). The biological role of poly-SUMO chains is still largely unknown in eukaryotes and few substrates have been identified. SUMO chain formation requires internal lysines within sumoylation consensus sites and is not required for viability in budding yeast during vegetative growth (Bylebyl et al., 2003). However, SUMO polymers play a structural role during meiosis in yeast and mitosis in mammalian cells (Cheng et al., 2006; Zhang et al., 2008). Moreover, the attachment of poly-SUMO chains to a substrate can promote its subsequent ubiquitylation and degradation, thereby acting as ubiquitylation signals in the turnover of SUMO targets (Ulrich, 2008; Geoffroy and Hay, 2009). We speculate that Velo acts likely in the deconjugation of poly-SUMO chains because of the sequence similarities to SENP7. However, roles for poly-SUMO chains in neurons and crosstalks between sumoylation and ubiquitination pathways during neuronal target selection remain to be determined.

Further elucidation of the mechanism by which Velo regulates PN dendrite and axon targeting requires identification of its target substrate(s). Because Velo-HA localizes to the nucleus, the potential substrate is likely a nuclear protein. Numerous studies have demonstrated a role for sumoylation regulating transcription (Seeler and Dejean, 2003; Verger et al., 2003). For example, the E3 SUMO ligase and transcriptional coregulator Protein Inhibitor of Activated Stat3 (Pias3) controls rod photoreceptor development and differentiation in the mouse retina by regulating transcription factors via sumoylation (Onishi et al., 2009, 2010). Furthermore, several transcription factors have been shown to regulate $\mathrm{PN}$ dendrite target selection when misexpressed or mutated (Komiyama et al., 2003; Komiyama and Luo, 2007; Spletter et al., 2007). Another likely set of substrates for Velo includes factors involved in chromosome organization and function. Indeed, it has recently been shown that SMC1, a cohesin subunit required for sister chromatid cohesion during mitosis and meiosis, and the chromatin remodeling factor Rpd3, a class 1 histone deacetylase (HDAC1) involved in chromatin integrity, play roles during PN targeting (Schuldiner et al., 2008; Tea et al., 2010). Future studies on candidate genes that exhibit similar neuronal targeting errors, together with biochemical and proteomic approaches, might uncover potential Velo substrates, and provide further insight into how sumoylation participates in the precise wiring of the olfactory circuit.

\section{References}

Bylebyl GR, Belichenko I, Johnson ES (2003) The SUMO isopeptidase Ulp2 prevents accumulation of SUMO chains in yeast. J Biol Chem 278:44113-44120. 
Cheng CH, Lo YH, Liang SS, Ti SC, Lin FM, Yeh CH, Huang HY, Wang TF (2006) SUMO modifications control assembly of synaptonemal complex and polycomplex in meiosis of Saccharomyces cerevisiae. Genes Dev 20:2067-2081.

Di Bacco A, Ouyang J, Lee HY, Catic A, Ploegh H, Gill G (2006) The SUMOspecific protease SENP5 is required for cell division. Mol Cell Biol 26:4489-4498.

Dorval V, Fraser PE (2007) SUMO on the road to neurodegeneration. Biochim Biophys Acta 1773:694-706.

Feligioni M, Nishimune A, Henley JM (2009) Protein SUMOylation modulates calcium influx and glutamate release from presynaptic terminals. Eur J Neurosci 29:1348-1356.

Geiss-Friedlander R, Melchior F (2007) Concepts in sumoylation: a decade on. Nat Rev Mol Cell Biol 8:947-956.

Geoffroy MC, Hay RT (2009) An additional role for SUMO in ubiquitinmediated proteolysis. Nat Rev Mol Cell Biol 10:564-568.

Hay RT (2007) SUMO-specific proteases: a twist in the tail. Trends Cell Biol $17: 370-376$

Hong W, Zhu H, Potter CJ, Barsh G, Kurusu M, Zinn K, Luo L (2009) Leucine-rich repeat transmembrane proteins instruct discrete dendrite targeting in an olfactory map. Nat Neurosci 12:1542-1550.

Jefferis GS, Marin EC, Stocker RF, Luo L (2001) Target neuron prespecification in the olfactory map of Drosophila. Nature 414:204-208.

Jefferis GS, Vyas RM, Berdnik D, Ramaekers A, Stocker RF, Tanaka NK, Ito K, Luo L (2004) Developmental origin of wiring specificity in the olfactory system of Drosophila. Development 131:117-130.

Jefferis GS, Potter CJ, Chan AM, Marin EC, Rohlfing T, Maurer CR Jr, Luo L (2007) Comprehensive maps of Drosophila higher olfactory centers: spatially segregated fruit and pheromone representation. Cell 128:1187-1203.

Johnson ES (2004) Protein modification by SUMO. Annu Rev Biochem 73:355-382.

Komiyama T, Luo L (2007) Intrinsic control of precise dendritic targeting by an ensemble of transcription factors. Curr Biol 17:278-285.

Komiyama T, Johnson WA, Luo L, Jefferis GS (2003) From lineage to wiring specificity: POU domain transcription factors control precise connections of Drosophila olfactory projection neurons. Cell 112:157-167.

Komiyama T, Sweeney LB, Schuldiner O, Garcia KC, Luo L (2007) Graded expression of semaphorin-1a cell-autonomously directs dendritic targeting of olfactory projection neurons. Cell 128:399-410.

Lee T, Luo L (1999) Mosaic analysis with a repressible cell marker for studies of gene function in neuronal morphogenesis. Neuron 22:451-461.

Li SJ, Hochstrasser M (1999) A new protease required for cell-cycle progression in yeast. Nature 398:246-251.

Li SJ, Hochstrasser M (2000) The yeast ULP2 (SMT4) gene encodes a novel protease specific for the ubiquitin-like Smt3 protein. Mol Cell Biol 20:2367-2377.

Lima CD, Reverter D (2008) Structure of the human SENP7 catalytic domain and poly-SUMO deconjugation activities for SENP6 and SENP7. J Biol Chem 283:32045-32055.

Marin EC, Jefferis GS, Komiyama T, Zhu H, Luo L (2002) Representation of the glomerular olfactory map in the Drosophila brain. Cell 109:243-255.

Martin S, Wilkinson KA, Nishimune A, Henley JM (2007) Emerging extranuclear roles of protein SUMOylation in neuronal function and dysfunction. Nat Rev Neurosci 8:948-959.

Mukhopadhyay D, Dasso M (2007) Modification in reverse: the SUMO proteases. Trends Biochem Sci 32:286-295.

Onishi A, Peng GH, Hsu C, Alexis U, Chen S, Blackshaw S (2009) Pias3dependent SUMOylation directs rod photoreceptor development. Neuron 61:234-246.

Onishi A, Peng GH, Chen S, Blackshaw S (2010) Pias3-dependent SUMOylation controls mammalian cone photoreceptor differentiation. Nat Neurosci 13:1059-1065.
Parks AL, Cook KR, Belvin M, Dompe NA, Fawcett R, Huppert K, Tan LR, Winter CG, Bogart KP, Deal JE, Deal-Herr ME, Grant D, Marcinko M, Miyazaki WY, Robertson S, Shaw KJ, Tabios M, Vysotskaia V, Zhao L, Andrade RS, et al. (2004) Systematic generation of high-resolution deletion coverage of the Drosophila melanogaster genome. Nat Genet 36:288-292.

Scheschonka A, Tang Z, Betz H (2007) Sumoylation in neurons: nuclear and synaptic roles? Trends Neurosci 30:85-91.

Schuldiner O, Berdnik D, Levy JM, Wu JS, Luginbuhl D, Gontang AC, Luo L (2008) piggyBac-based mosaic screen identifies a postmitotic function for cohesin in regulating developmental axon pruning. Dev Cell 14:227-238.

Seeler JS, Dejean A (2003) Nuclear and unclear functions of SUMO. Nat Rev Mol Cell Biol 4:690-699.

Shalizi A, Gaudillière B, Yuan Z, Stegmüller J, Shirogane T, Ge Q, Tan Y, Schulman B, Harper JW, Bonni A (2006) A calcium-regulated MEF2 sumoylation switch controls postsynaptic differentiation. Science 311:1012-1017.

Shen LN, Geoffroy MC, Jaffray EG, Hay RT (2009) Characterization of SENP7, a SUMO-2/3-specific isopeptidase. Biochem J 421:223-230.

Smith M, Bhaskar V, Fernandez J, Courey AJ (2004) Drosophila Ulp1, a nuclear pore-associated SUMO protease, prevents accumulation of cytoplasmic SUMO conjugates. J Biol Chem 279:43805-43814.

Spletter ML, Liu J, Su H, Giniger E, Komiyama T, Quake S, Luo L (2007) Lola regulates Drosophila olfactory projection neuron identity and targeting specificity. Neural Develop 2:14.

Steffan JS, Agrawal N, Pallos J, Rockabrand E, Trotman LC, Slepko N, Illes K, Lukacsovich T, Zhu YZ, Cattaneo E, Pandolfi PP, Thompson LM, Marsh JL (2004) SUMO modification of Huntingtin and Huntington's disease pathology. Science 304:100-104.

Stocker RF, Heimbeck G, Gendre N, de Belle JS (1997) Neuroblast ablation in Drosophila P[GAL4] lines reveals origins of olfactory interneurons. J Neurobiol 32:443-456.

Sun X, Huang L, Van Doren MR, Tanda S (2003) Isolation of amorphic alleles of the lesswright gene by P-element mediated male recombination in Drosophila melanogaster. Dros Inf Serv 86:79-83.

Tea JS, Chihara T, Luo L (2010) Histone deacetylase Rpd3 regulates olfactory projection neuron dendrite targeting via the transcription factor Prospero. J Neurosci 30:9939-9946.

Thibault ST, Singer MA, Miyazaki WY, Milash B, Dompe NA, Singh CM, Buchholz R, Demsky M, Fawcett R, Francis-Lang HL, Ryner L, Cheung LM, Chong A, Erickson C, Fisher WW, Greer K, Hartouni SR, Howie E, Jakkula L, Joo D, et al. (2004) A complementary transposon tool kit for Drosophila melanogaster using P and piggyBac. Nat Genet 36:283-287.

Ulrich HD (2008) The fast-growing business of SUMO chains. Mol Cell 32:301-305.

Verger A, Perdomo J, Crossley M (2003) Modification with SUMO. A role in transcriptional regulation. EMBO Rep 4:137-142.

Wilkinson KA, Henley JM (2010) Mechanisms, regulation and consequences of protein SUMOylation. Biochem J 428:133-145.

Wilkinson KA, Nakamura Y, Henley JM (2010) Targets and consequences of protein SUMOylation in neurons. Brain Res Rev 64:195-212.

Wong AM, Wang JW, Axel R (2002) Spatial representation of the glomerular map in the Drosophila protocerebrum. Cell 109:229-241.

Wu JS, Luo L (2006a) A protocol for mosaic analysis with a repressible cell marker (MARCM) in Drosophila. Nat Protoc 1:2583-2589.

Wu JS, Luo L (2006b) A protocol for dissecting Drosophila melanogaster brains for live imaging or immunostaining. Nat Protoc 1:2110-2115.

Zhang XD, Goeres J, Zhang H, Yen TJ, Porter AC, Matunis MJ (2008) SUMO-2/3 modification and binding regulate the association of CENP-E with kinetochores and progression through mitosis. Mol Cell 29:729741 . 